\title{
Vitamin A Homeostasis in the Diabetic Rat
}

\author{
Andrew T.C. Tsin, ${ }^{1, *}$ Brenda W. GRIfFIN, ${ }^{2}$ Nathan L. MATA, ${ }^{1}$ \\ Hing-Sing YU, ${ }^{1}$ Gary W. Williams, ${ }^{2}$ Julie Y. CRIDER, ${ }^{2}$ \\ and Michael L. CHANDLER ${ }^{2}$ \\ ${ }^{1}$ Division of Life Sciences, The University of Texas at San Antonio, San Antonio, \\ Texas, U.S.A. \\ ${ }^{2}$ Alcon Laboratories, Inc., Fort Worth, Texas, U.S.A.
}

(Received February 18, 1993)

\begin{abstract}
Summary The concentrations of vitamin A (retinol) and retinyl ester in the plasma and liver of normal and diabetic rats were measured by HPLC (high-performance liquid chromatography). Diabetic rats had severe hyperglycemia, induced by a single streptozotocin injection 5 weeks prior to sampling. In the normal rats, plasma retinyl palmitate was very low, and the level was increased 10 -fold by diabetes. Detailed timecourse studies showed that rats became hyperglycemic within $48 \mathrm{~h}$ of streptozotocin injection, yet the plasma retinyl palmitate level was not elevated until some three weeks later. Severe diabetes did not significantly influence plasma retinol; however, free retinol in the liver was elevated within 10 days of initiation of the disease and continued to increase for the duration of the study. These results show that streptozotocin-induced diabetes significantly alters the concentrations of hepatic retinol and plasma retinyl ester. The biochemical mechanism(s) of this altered vitamin A homeostasis in diabetes and its possible relationship to tissue pathogenesis are not known at present.
\end{abstract}

Key Words: diabetes, hyperglycemia, streptozotocin, vitamin A, retinyl ester

Diabetes refers to metabolic disorders associated with hyperglycemia. Type I diabetes (juvenile-onset, insulin-dependent) is characterized by insulin deficiency; whereas in Type II diabetes (late-onset), the insulin level may be normal (or even elevated) but insulin is not effective in target organs. Various animal models have been developed to study the metabolic perturbations and underlying causes of diabetes mellitus $[1,2]$. In most of these experimental models, diabetes is induced

\footnotetext{
*To whom correspondence should be addressed.
} 
directly by environmental insults (viruses and chemical agents) which, in some cases, may interact with a susceptible genetic profile. Spontaneously diabetic animal models have also been characterized.

Many metabolic anomalies are associated with diabetes. The cumulative, chronic effects of this metabolic disease lead eventually to debilitating functional and morphological alterations, such as nephro-, neuro-, and retino-pathy [2]. Cardiovascular responses to diabetes include an increase in plasma lipoproteins, atherosclerosis, hypertension, and cardiomyopathy [3]. In large animals such as dogs and primates, including humans, diabetic retinopathy can progress to the proliferative stage, which is associated with neovascularization of retinal vessels, vitreous hemorrhage, retinal detachment, and visual impairment $[4,5]$.

Although changes in many metabolic parameters have been measured in diabetic animals and human patients, the effect of diabetes on vitamin A metabolism has not been fully documented. Bowles reported a decrease in hepatic storage of vitamin A in rat in response to insulin [6], and Mosenthal and Laughlin suggested that diabetic children had lower levels of plasma vitamin A [7]. Recently, Basu et al. [8] and Krempf et al. [9] reported lower levels of plasma retinol and retinol binding protein (RBP) in patients with Type I diabetes. Additionally, Wako et al. [10] found higher retinyl ester concentrations in the plasma of patients with Type II diabetes. Almost all of these plasma retinyl esters were associated with high-density lipoprotein (HDL), low-density lipoprotein (LDL), and larger molecular size lipoproteins. A recent study showed that plasma retinyl ester was elevated in postprandial Type II diabetic patients but not in fasting (overnight) patients [11]. In the present study, we investigated plasma and hepatic levels of retinoids in normal and diabetic rats. Salient features of our results are as follows: 1) A marked increase in the plasma retinyl ester level (but not plasma retinol) was seen in response to diabetes. This change occurred approximately 2 weeks subsequent to the onset of hyperglycemia. 2) Diabetes induced an immediate elevation of hepatic retinol, which steadily increased to a level nearly 5-times greater than the control value 37 days post-streptozotocin injection.

Because retinoids are very potent modulators of cell growth and differentiation [12], a significant alteration in their circulatory levels, together with a change in insulin and glucose homeostasis, could lead to proliferative changes in target organs as exemplified in diabetic retinopathy. Thus, diabetes-induced alteration in vitamin A metabolism is likely to play an important role in the pathogenesis of different susceptible tissues. The identification of these tissues as well as the investigation into the underlying cell and molecular events responsive to retinoids remains to be carried out.

\section{MATERIALS AND METHODS}

Animal model of diabetes. All experiments were approved by the appropri- 
ate animal use and care committees and were conducted in strict accordance with the ARVO (Association for Research in Vision and Ophthalmology) Resolution on the Use of Animals in Research. The experimental details of this animal model have been previously described [13].

Sprague Dawley rats were randomly divided into two groups of control and treated animals. The treated group received a single i.v. injection (tail vein) of streptozotocin $(50 \mathrm{mg} / \mathrm{kg})$ dissolved in $0.01 \mathrm{M}$ citrate buffer, $\mathrm{pH} 4.5$. Blood samples were taken by venipuncture. Plasma glucose concentrations were determined from blood samples by the glucose oxidase method (Sigma Chemical Co., St. Louis, MO). Both groups were fed ad libitum with Purina's Certified Autoclavable Rodent Diet No. 5014 (20\% protein, 10\% fat, 70\% carbohydrate) with a vitamin A content of $37.4 \mathrm{IU} / \mathrm{g}$. Food consumption in the treated group was typically 3 -times greater than that in the control throughout the course of the study $(9 \mathrm{~g} / \mathrm{day} / 100 \mathrm{~g}$ body weight vs. $3 \mathrm{~g} / \mathrm{day} / 100 \mathrm{~g}$ body weight). The animals were fasted overnight prior to sacrifice (by $\mathrm{CO}_{2}$ asphyxiation at regular intervals: 2, 9, 23, 37 days post-injection, $n=8$ ). Blood was collected by cardiac puncture into a heparinized tube. After centrifugation, the plasma was separated and frozen. Whole livers were removed, weighed, placed in individual plastic bags, and frozen with liquid $\mathrm{N}_{2}$. Samples were stored at $-80^{\circ} \mathrm{C}$ until analyzed.

Chemicals and solvents. Ethyl alcohol USP was purchased from Aaper Alcohol and Chemical Co., Shelbyville, KY. ChromAR HPLC grade $n$-hexane and 1,4-dioxane were purchased from Mallinckrodt, Paris, KY. Retinol, retinyl palmitate, and streptozotocin were obtained from Sigma Chemical Co. Alumina (basic aluminum oxide, activity grade I) was obtained from Universal Scientific Inc., Atlanta, GA. Ultra high purity $\mathrm{N}_{2}$ was purchased from TriGas Industrial Gases, Irving, TX.

High-performance liquid chromatography. HPLC Unit No. 1 consisted of a Kontron T-414 pump, a Rheodyne 7125 injection valve, a Beckman $160 \mathrm{UV}$ detector, a Waters "Resolve" $15-\mathrm{cm} 5-\mu \mathrm{m}$ silica HPLC column (eluted with $0.2 \%$ dioxane in $n$-hexane to analyze retinyl esters), a Hewlett-Packard 3390A integrator, and a Hamilton 710-LT $50 \mu 1$ injection syringe. HPLC Unit No. 2 consisted of a Beckman 112 Solvent Delivery Module, a Rheodyne 7125 injection valve, a Beckman 160 UV detector, a Waters "Resolve" $15-\mathrm{cm}$ 5- $\mu \mathrm{m}$ silica HPLC column (eluted with $10 \%$ dioxane in $n$-hexane to analyze retinols), a Hewlett-Packard 3390A integrator, and a Hamilton 710-LT $50 \mu$ injection syringe.

Estimation of retinoids in blood plasma. Tubes containing blood plasma were thawed and $0.5 \mathrm{ml}$ from each sample removed to $16 \times 150 \mathrm{~mm}$ culture tubes. To each tube, $0.5 \mathrm{ml}$ of ethyl alcohol was added and mixed. Each tube was then extracted four times with $2 \mathrm{ml} n$-hexane, and the solvent of the combined tissue extract was removed by a stream of $\mathrm{N}_{2}$. The residue was dissolved in $1 \mathrm{ml}$ of $0.5 \%$ dioxane in $n$-hexane and loaded onto an alumina column (5\% water deactivated; $1 \times 2 \mathrm{~cm})$. Retinyl ester was recovered by elution with $8 \mathrm{ml}$ of $0.5 \%$ dioxane in $n$-hexane, and retinol was then collected by elution with $8 \mathrm{ml}$ of $10 \%$ dioxane in 
$n$-hexane. These fractions were evaporated to dryness by $\mathrm{N}_{2}$. The residue was redissolved in a measured volume of the HPLC mobile phase, and a 5-40- $\mu 1$ portion injected into the appropriate HPLC Unit (i.e., retinyl ester: Unit No. 1; retinol: Unit No. 2). Retinoid concentration was determined by comparison of sample peak areas with calibration curves derived from the use of authentic standards. Detection limit was estimated at one picomol.

Estimation of retinoids in liver. Approximately $1 \mathrm{~g}$ of liver was ground with a mortar and pestle in anhydrous sodium sulfate until a fine powder was achieved. The powder was then transferred to a $16 \times 150 \mathrm{~mm}$ culture tube. Powdered liver tissue was extracted with $8 \mathrm{ml}$ of acetone, then twice more with $3 \mathrm{ml}$ of acetone; and the solvent of the combined tissue extract was removed by a stream of $\mathrm{N}_{2}$. The residue was dissolved in $1 \mathrm{ml}$ of $0.5 \%$ dioxane in $n$-hexane and introduced into an alumina column to separate retinyl esters from retinols before HPLC analyses.

Statistics. Student's $t$-test was used to compare control and experimental group means. Relative significance of temporal changes in retinyl palmitate, retinol, and glucose between the groups was assessed by two-way analysis of variance (ANOVA). Pairwise comparisons by Dunnett's tests were then performed to locate time-points at which parameters of the treated animals were significantly different from those of the control group. Statistical analyses were carried out according to methods described by Meyers [14].

\section{RESULTS}

Periodic measurement of plasma glucose in normal and streptozotocin-treated animals revealed a significant elevation in the experimental group within $48 \mathrm{~h}$ of

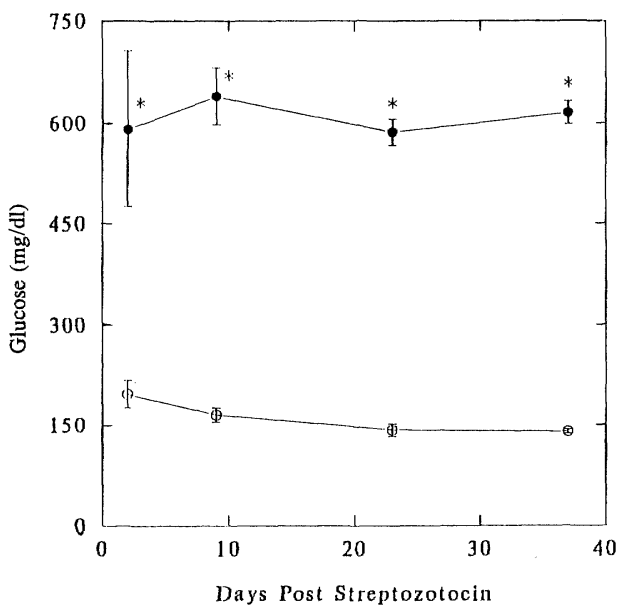

Fig. 1. Analysis of plasma glucose concentration in normal and diabetic animals. Blood samples taken from normal (open circles) and diabetic (filled circles) animals were assayed for glucose concentration according to the glucose oxidase method. 
injection of the streptozotocin. Glucose levels in the experimental group were approximately 4-times greater than control values and were maintained throughout the course of the study, thus indicating the efficacy of streptozotocin to induce diabetes (Fig. 1). HPLC analysis of retinoids extracted from plasma and liver of control and diabetic groups showed that retinyl palmitate and retinol were the only major components in the respective ester and alcohol fractions collected from the alumina column. In HPLC Unit No. 1, retinyl ester from blood and liver samples had a retention time of $4.9 \mathrm{~min}$ and coeluted with authentic all-transretinyl palmitate. Beta carotene, which has a retention time of $1.8 \mathrm{~min}$ in this elution system, was not found in any significant quantity. The identity of the retinyl ester in the blood sample was further elucidated by collection of the retinyl
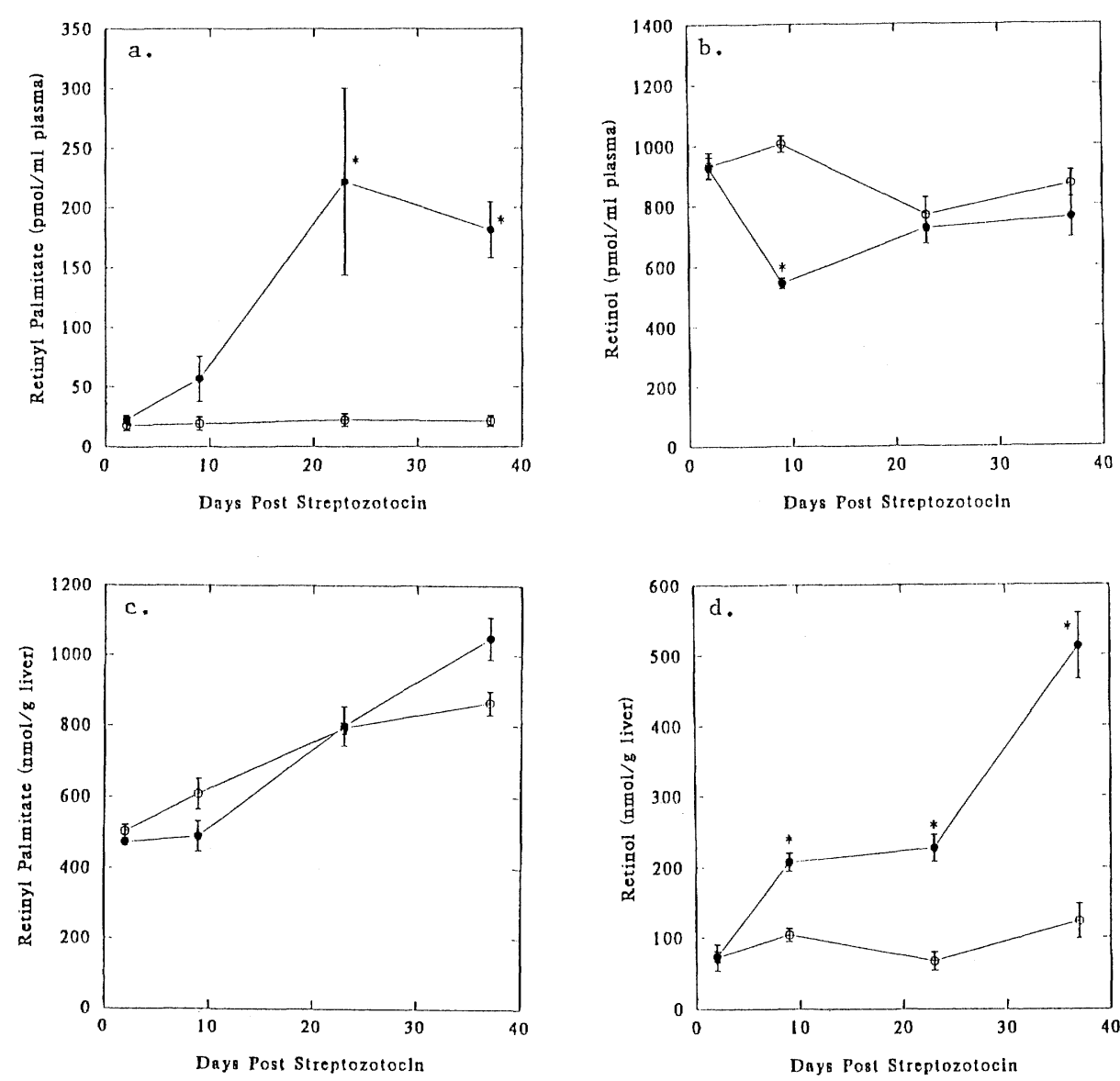

Fig. 2. Alterations in circulating retinyl palmitate (a) and retinol (b), and hepatic retinyl palmitate (c) and retinol (d) with time. Rats were injected with streptozotocin and samples were taken at $2,9,23$, and 37 days post-injection. Open circles, control rats; filled circles, diabetic rats. 
ester from the HPLC eluant and analysis of its absorption spectrum by use of a spectrophotometer. In HPLC Unit No. 2, retinol from blood and liver samples had a retention time of $5.3 \mathrm{~min}$ and coeluted with authentic all-trans-retinol.

Temporal changes in retinyl palmitate and retinol levels in plasma of normal and diabetic animals are illustrated in Fig. 2(a) and 2(b), respectively. Plasma retinyl palmitate levels were similar between the two groups on the 2 nd and 9 th day post-injection (Fig. 2(a)). However, at subsequent sampling time points, retinyl palmitate in the diabetic animals was found to be significantly higher than the control group level. Meanwhile, no difference was noted in blood retinol between the two treatment groups throughout the test period, except at the 9-day time point (Fig. 2(b)). During the 37-day period of study, the retinyl palmitate concentration in the liver remained similar between the two groups (Fig. 2(c)). However, a consistent and significant increase in hepatic retinol was observed in the diabetic animals relative to the level in the untreated control animals (Fig. 2(d)). Free retinol in diabetic liver was typically 5-times greater than the control value 37 days post-streptozotocin.

\section{DISCUSSION}

In the circulatory system, vitamin $\mathrm{A}$ is transported as all-trans-retinol bound to serum RBP [15]. A few exceptions to this general rule exist. For example, a significant level of plasma retinyl ester has been noted during hypervitaminosis A in rats [16] and humans [17], and in patients with Type II diabetes [9, 10]. Additionally, retinyl esters are the major form of vitamin A in the blood of normal dogs and other carnivores [18]. In all of these cases, retinyl ester circulates in the blood bound to lipoproteins $[9,10,16,19]$. Typically, lipoproteins solubilize highly hydrophobic lipids and regulate lipid movement via receptor-mediated endocytosis. Because chylomicron remnants and low-density lipoproteins contain apolipoprotein E, they may interact with the low-density lipoprotein receptor (apo $\mathrm{B} / \mathrm{E}$ receptor [20]) to facilitate retinyl ester delivery to extrahepatic target cells and tissues.

In the present study, we found an elevation of plasma retinyl ester levels in streptozotocin-treated adult Sprague Dawley rats. Increased plasma retinyl esters were observed about 2 weeks after the onset of severe hyperglycemia and were maintained throughout the 37-day experimental period. As circulating retinyl ester exists only minimally in this species, the relative increase in plasma retinyl ester was quite significant (i.e., nearly ten-fold). Nevertheless, these elevated retinyl ester levels remained at concentrations several times lower than those of the corresponding plasma retinol, which were similar between the treated and control groups. Histological studies of streptozotocin-induced insulitis have demonstrated that streptozotocin does not entirely ablate pancreatic $\beta$-cells at the concentrations used in the present study [21]. Therefore, the elevation of retinyl esters observed in the diabetic rats may not be solely attributable to complete absence of insulin. 
It has been demonstrated that conditions such as hypervitaminosis $\mathrm{A}$ and insulin-dependent diabetes mellitus result in significant decreases in RBP synthesis and RBP secretion from the liver $[8,16]$. Consequently, retinol may accumulate in the liver as its carrier protein is deficient. When one considers this and the fact that diabetic rats typically consume more food than their controls (up to 3 times more in the present study), increased levels of retinol in diabetic liver are not entirely surprising. Therefore, in the diabetic state, mobilization of vitamin A from the liver to extrahepatic target tissues may be accomplished by an alternative mechanism of delivery (e.g., HDL and LDL transport of stored retinyl esters). Circulating retinyl esters (mobilized via the low-density lipoprotein pathway) would ultimately be internalized and then hydrolyzed by lysosomes, thereby liberating all-trans-retinol. The possibility that vitamin A metabolism may be somehow linked to cholesterol and lipid metabolism in the diabetic state will require further study.

Retinol bound to RBP has been considered to be the physiologically important form of vitamin A transported in plasma and utilized by tissues, which convert it to the biologically more potent retinoic acid [22]. The potential physiologic significance of circulating retinyl esters has generally been neglected because the normal plasma levels in most species are very low. However, there are a few recent reports suggesting that retinyl esters may play more important roles than previously considered: 1) dogs and most carnivores normally have high plasma retinyl ester levels compared with other species [18]; and 2) retinyl ester in chylomicron remnants is the most effective species of vitamin A for regulating the growth and differentiation of leukemic cells [23]. Wathne et al. demonstrated that both retinol bound to RBP and retinyl ester bound to chylomicrons inhibited growth of myeloid leukemic cells but that only retinyl ester in its physiologic "vehicle," and at physiologic concentrations, could also induce differentiation of these cells.

It is also reasonable to suggest that diabetes alters retinoid metabolism through decreases in hepatic retinyl ester synthesis and hydrolysis. Because many of the biochemical mechanisms underlying release, transport, and utilization of retinyl esters have not been elucidated, the detailed relationship between diabetes and retinoid homeostasis is unclear at present. Nevertheless, our data are in good agreement with those recently published by Basu et al. [24] on the effect of streptozotocin-induced diabetes on plasma and hepatic retinol levels in rats. Moreover, the critical reaction in the visual cycle that eluded investigators until very recently, isomerization of all-trans-retinol to 11-cis-retinol in RPE cells, involves formation of retinyl esters as energetic intermediates [25]. Whether extracellular retinyl esters could be taken up and utilized as substrates for this reaction under abnormal (e.g., diabetic) conditions is not known. Although the available data are limited, they suggest that the specific cellular responses to vitamin A depend upon both the retinoid species and the mechanism by which it enters the cell and that retinyl esters may play an important role in the biological 
effects of vitamin A.

In summary, this is the first report to demonstrate the marked alteration in plasma retinyl ester concentration in diabetic rats. The relationship among glucose, insulin, retinyl ester, and retinol in circulation is obviously very complex. As an example of this complexity, there is evidence obtained in rats that insulin causes depletion of hepatic vitamin A stores [6] and also that vitamin A is required for normal insulin secretion [26]. Moreover, there are species differences and aging influences on vitamin A transport and homeostasis that greatly confound attempts to extrapolate data from well-controlled animal studies to humans. Based on our data and information available in the literature on diabetic dogs [18, 27] and diabetic humans $[1,10]$ chronic alteration of circulatory retinyl esters may contribute to the risk of proliferative pathology in a tissue such as the retina, which has unique requirements for retinoids. The functions of other tissues of diabetic humans may also be compromised by alterations in vitamin A homeostasis. Additional studies will be required to elucidate the complex inter-relationships between vitamin A-metabolizing activities, plasma retinyl ester, and other plasma components such as glucose, lipids, and insulin under the diabetic condition.

We thank Dr. T.K. Basu for review of the manuscript and Douglas Malsbury for technical support. This research was supported by grants from the NIH and the San Antonio Area Foundation.

\section{REFERENCES}

1. Grodsky, G.M., Anderson, C.E., Coleman, D.L., Craighead, J.E., Gerritsen, G.C., Hansen, C.T., Herberg, L., Howard, C.F., Jr., Lernmark, A., Matschinsky, F.M., Rayfield, E., Riley, W.J., and Rossini, A.A. (1982): Metabolic and underlying causes of diabetes mellitus. Diabetes, 31, Suppl. 1, 45-53.

2. Shafrir, E., and Renold, A.E. (eds.) (1988): Frontiers in Diabetes Research, John Libbey, London.

3. Chobanian, A.V., Arquilla, E.R., Clarkson, T.B., Eder, H.A., Howard, C.F., Jr., Regan, T.J., and Williamson, J.R. (1982): Cardiovascular complications. Diabetes, 31, Suppl. 1, 5464.

4. Engerman, R., Finkelstein, D., Aguirre, G., Diddie, K.R., Fox, R.R., Frank, R.N., and Varma, S.D. (1982): Ocular complications. Diabetes, 31, Suppl. 1, 82-88.

5. Chakrabarti, S., and Sima, A.A.F. (1988): Frontiers in Diabetes Research, John Libbey, London.

6. Bowles, W.H. (1967): Influence of insulin on liver vitamin A in rats. Diabetes, 16, 704-707.

7. Mosenthal, H.O., and Laughlin, W.C. (1944): Vitamin A, B and C in diabetic children. Arch. Intern. Med., 73, 391-396.

8. Basu, T.K., Tze, W.J., and Leichter, J. (1989): Serum vitamin A and retinol-binding protein in patients with insulin-dependent diabetes mellitus. Am. J. Clin. Nutr., 50, 329-331.

9. Krempf, M., Ranganathan, S., Ritz, P., Morin, M., and Charbonnel, B. (1991): Plasma vitamin $\mathrm{A}$ and $\mathrm{E}$ in Type I (insulin-dependent) and Type II (non-insulin-dependent) adult diabetic patients. Int. J. Vitam. Nutr. Res., 61, 38-42.

10. Wako, Y., Suzuki, K., Goto, Y., and Kimura, S. (1986): Vitamin A transport in plasma of diabetic patients. Tohoku J. Exp. Med., 149, 133-143. 
11. Lewis, G.F., O’Meara, N.M., Soltys, P.A., Blackman, J.D., Iverius, P.H., Pugh, W.L., Getz, G.S., and Polonsky, K.S. (1991): Fasting hypertriglyceridemia in noninsulin-dependent diabetes mellitus is an important predictor of postprandial lipid and lipoprotein abnormalities. J. Clin. Endocrinol. Metab., 72, 934-944.

12. Sporn, M.B., Roberts, A.B., and Goodman, D.S. (eds.) (1984): The Retinoids, Academic Press, New York.

13. Griffin, B.W., McNatt, L.G., Chandler, M.L., and York, B.M. (1987): Effects of two new aldose reductase inhibitors, AL-1567 and AL-1576, in diabetic rats. Metabolism, 36, 486490.

14. Meyers, J.L. (1979): Fundamentals of Experimental Design (3rd ed.), Allyn and Bacon, Inc., Boston.

15. Peterson, P.A., and Beggard, I. (1971): Isolation and properties of a human retinoltransporting protein. J. Biol. Chem., 246, 25-33.

16. Mallia, A.K., Smith, J.E., and Goodman, D.S. (1975): Metabolism of retinol-binding protein and vitamin A during hypervitaminosis A in the rat. J. Lipid Res., 16, 180-188.

17. Smith, F.R., and Goodman, D.S. (1976): Vitamin A transport in human vitamin A toxicity. N. Engl. J. Med., 294, 805-808.

18. Schweigert, F.J., Ryder, O.A., Rambeck, W.A., and Zucker, H. (1990): The majority of vitamin A is transported as retinyl esters in the blood of most carnivores. Comp. Biochem. Physiol., 95A, 573-578.

19. Wilson, D.E., Hejazi, J., Elstad, N.L., Chan, I.-F., Gleeson, J.M., and Iverius, P.-H. (1987): Novel aspect of vitamin A metabolism in the dog: Distribution of lipoprotein retinyl esters in vitamin A-deprived and cholesterol-fed animals. Biochim. Biophys. Acta, 922, 247-258.

20. Floren, C.H., Albers, J.J., Kudchodker, B.J., and Bierman, E.L. (1981): Receptor-dependent uptake of human chylomicron remnants by cultured skin fibroblasts. J. Biol. Chem., 256, 425-433.

21. Iwakiri, R., and Nagafuchi, S. (1989): Inhibition of streptozotocin induced insulitis and diabetes with lobenzarit in CD-1 mice. Diabetes, 38, 558-561.

22. Blomhoff, R., Skrede, B., and Norum, K.R. (1990): Uptake of chylomicron remnant retinyl ester via the low density lipoprotein receptor: Implications for the role of vitamin A as a possible preventive for some forms of cancer. J. Intern. Med., 228, 207-210.

23. Wathne, K.-O., Norum, K.R., Smeland, E., and Blomhoff, R. (1988): Retinol bound to physiological carrier molecules regulates growth and differentiation of myeloid leukemic cells. J. Biol. Chem., 263, 8691-8695.

24. Basu, T.K., Leichter, J., and McNeill, J.H. (1990): Plasma and liver vitamin A concentrations in streptozotocin diabetic rats. Nutr. Res., 10, 421-427.

25. Rando, R.R., Bernstein, P.S., and Barry, R.J. (1991): New insight into the visual cycle, in Progress in Retinal Research, Vol. 10, ed. by Osborne, N.N. and Chader, G.J., Pergamon Press, New York, pp. 161-178.

26. Chertow, B.S., Blaner, W.S., Baranetsky, N.G., Aivitz, W.I., Cordle, M.B., Thompson, D., and Meda, P. (1987): Effects of vitamin A deficiency and repletion on rat insulin secretion in vivo and in vitro from isolated islets. J. Clin. Invest., 79, 163-169.

27. Engerman, R.L., and Bloodworth, J.M. (1965): Experimental diabetic retinopathy in dogs. Arch. Ophthalmol., 73, 205-210. 